\title{
Prevalence of coronary heart disease among Tehran adults: Tehran Lipid and Glucose Study
}

F. Hadaegh, ${ }^{1}$ H. Harati, ${ }^{1}$ A. Ghanbarian ${ }^{1}$ and F. Azizi ${ }^{1}$

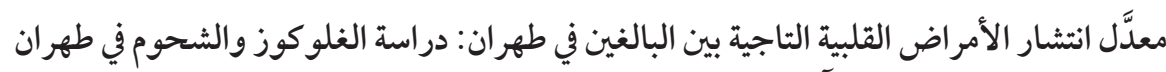

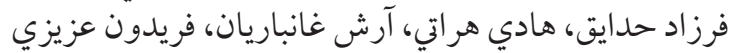

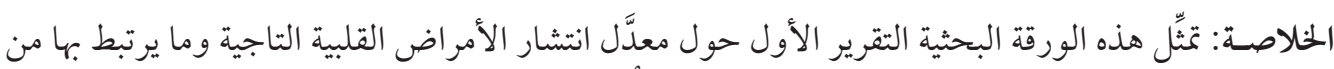

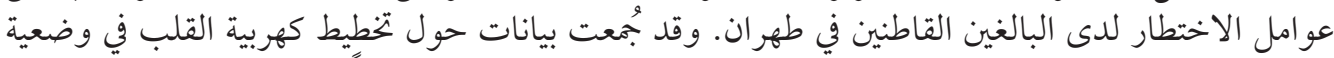

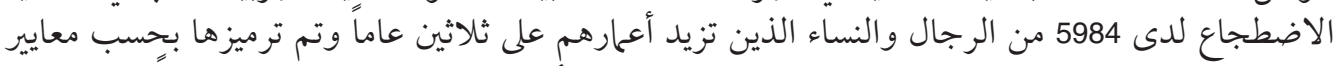

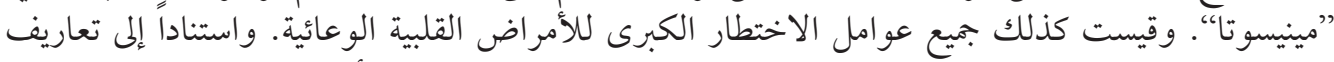

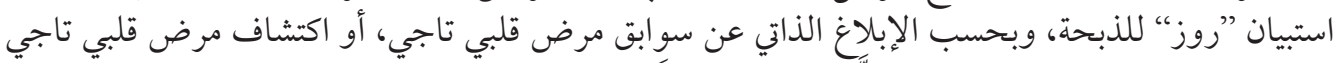

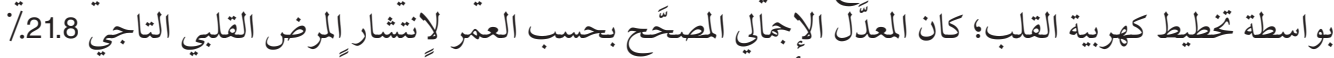

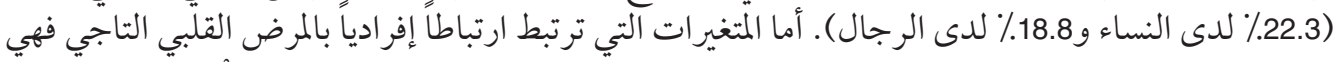

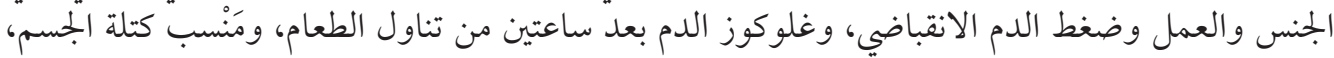

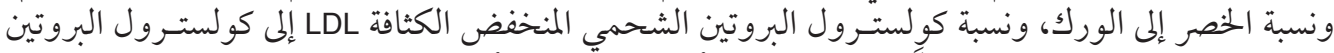

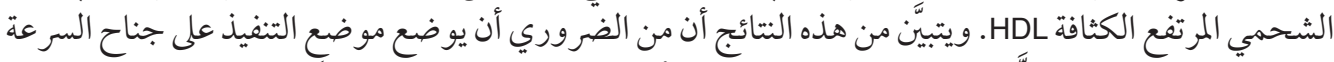

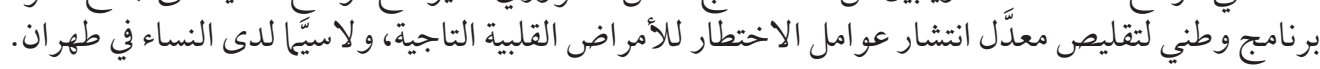

ABSTRACT This study is the first report on the prevalence of coronary heart disease (CHD) and its associated risk factors in adult residents of Tehran. Standard supine ECG data were collected for 5984 men and women aged $\geq 30$ years and coded by Minnesota criteria. All major cardiovascular risk factors were also measured. Based on Rose angina, self-reported history of CHD or ECG-defined CHD, the aged-adjusted prevalence of CHD was $21.8 \%$ (22.3\% in women and $18.8 \%$ in men). Variables independently associated with CHD were female sex, age, systolic blood pressure, 2-hour postprandial glucose, body mass index, waist-to-hip ratio and LDL/HDL cholesterol ratio.

\footnotetext{
Prévalence des cardiopathies coronariennes chez les adultes à Téhéran : étude sur les lipides et le glucose

RÉSUMÉ II s'agit de la première étude consacrée à la prévalence des cardiopathies coronariennes $(\mathrm{CPC})$ et aux facteurs de risque associés chez les adultes vivant à Téhéran. Un électrocardiogramme standard a été réalisé en position allongée sur 5984 hommes et femmes âgés de 30 ans et plus et les résultats ont été recueillis et codés selon les critères du Minnesota. Les principaux facteurs de risque cardiovasculaire ont également été mesurés. La prévalence ajustée sur l'âge des CPC, calculée sur la base de l'angor détecté grâce au questionnaire de Rose, des antécédents autodéclarés de CPC ou des CPC révélées par l'ECG, était globalement de $21,8 \%$ (22,3\% chez les femmes et $18,8 \%$ chez les hommes). Les variables indépendamment associées aux CPC étaient le sexe féminin, l'âge, la pression artérielle systolique, la glycémie postprandiale deux heures après un repas, l'indice de masse corporelle, le rapport tour de taille/tour de hanches et le rapport cholestérol LDL/HDL.
}

${ }^{1}$ Prevention of Metabolic Disorders Research Centre, Research Institute of Endocrine Sciences, Shaheed Beheshti University of Medical Sciences, Tehran, Islamic Republic of Iran (Correspondence to F. Azizi: azizi@erc.ac.ir).

Received: 22/01/06; accepted: 27/08/06

المجلة الصحية لشرق المتوسط، منظمة الصحة العالمية، المجلد الخامس عشر، العلد (، 9.9 


\section{Introduction}

Coronary heart disease (CHD) is one of the most common causes of morbidity and mortality in different communities $[1,2]$. In the United States of America, CHD is the leading cause of death in adults, accounting for approximately one-third of all deaths in people over the age of 35 years [3]. Despite the lack of accurate data, there is some evidence to indicate that CHD is increasing in magnitude in the Islamic Republic of Iran. While age-adjusted mortality from CHD is gradually falling in developed countries $[2,4]$, the rate has increased by $20 \%-45 \%$ in the Islamic Republic of Iran [5,6]. It seems likely that changing lifestyles such as high consumption of processed foods rich in saturated fat and a low level of physical activity along with the rising prevalence of obesity and type 2 diabetes are leading to a progressive increase in the prevalence of cardiovascular (CVD) risk factors and CHD in developing countries $[7,8]$.

Studies on the adult population of Tehran show a high prevalence of metabolic syndrome and CVD risk factors, particularly hypertension, high total cholesterol (TC) and low high-density lipoprotein cholesterol (HDL-C) and high waist-to-hip ratio (WHR) [9-11]. This study was conducted to determine the prevalence of CHD in Tehran from 3 measures - the Rose questionnaire on angina pectoris, self-reported previous medical history and Minnesota coding of a 12-lead resting electrocardiogram (ECG) and to identify its associated risk factors.

\section{Methods}

\section{Sample}

The Tehran Lipid and Glucose Study is a longitudinal study, the first phase of which was a cross-sectional or baseline examination survey from 1999 to 2001. It was de- signed to estimate the prevalence of CVD risk factors in a representative sample of an Iranian urban population selected by random cluster sampling from district no. 13 of Tehran. Details of the rationale and design have been published elsewhere [12]. There were 8071 individuals aged $\geq 30$ years who completed the baseline survey and whose data were used for the current study.

\section{Data collection}

The data were collected by means of interviews, with completion of a questionnaire for demographic data and CHD history, physical examination for blood pressure, pulse rate, ECG and anthropometrical measures and laboratory measurements for lipid and glucose profiles. The data collected were stored directly in a computer database [13].

\section{Coronary heart disease}

For this study, CHD was defined as symptoms of angina pectoris based on the Rose angina questionnaire or self-reported positive history of CHD or ECG positive for CHD. The prevalence of CHD was estimated after application of each individual criterion and also after combination of these 3 individual measures.

Using a Farsi language version of the Rose questionnaire, history of any chest pain was assessed during the interviews. Rose angina score was measured for participants who had chest pain during exertion. This pain forces the person to stop and goes away in less than 10 minutes when he/she stops. If present, the pain is situated over the anterior or left lateral sternum or radiates to the left arm [14].

Self-reported history of CHD was defined as a positive answer at the time of the interview to the question as to whether the patient had ever had a prior diagnosis of CHD by a physician. 
A 12-lead resting ECG was recorded for each individual $\geq 30$ years in the Tehran Lipid and Glucose population by 2 trained and qualified technicians according to a standard recording protocol developed by the School of Public Health, the University of Minnesota using a PC-ECG 1200 machine [15]. Two qualified physicians coded the ECGs independently according to the Minnesota codes using a measuring loupe specially manufactured by University of Minnesota [15]. To assure the quality, a third qualified physician recoded $10 \%$ of ECGs and all the data were double-entered. The population was categorized into 3 groups (probable CHD, possible CHD and non-CHD) on the basis of ECG findings and Whitehall criteria [16]. Minnesota codes of 1.1-1.2 were considered as probable CHD and codes 1.3, $4.1-4.4,5.1-5.3$ or 7.1 as possible CHD [16]. In our analysis, we considered both probable and possible CHD as a single definition of ECG-defined CHD.

\section{Clinical data}

The details of data collection have already been described [10]. This included systolic blood pressure (SBP), diastolic blood pressure (DBP), weight, height and waist circumference to calculate WHR, waist-toheight ratio (WHeR) and body mass index (BMI).

A blood sample was taken after 12-14 hours overnight fasting for biochemical measurements. Blood samples were taken in a sitting position according to the standard protocol and centrifuged within 30-45 min of collection. All blood analyses were done at the Tehran Lipid and Glucose Study research laboratory on the day of blood collection.

For the oral glucose tolerance test, $82.5 \mathrm{~g}$ glucose monohydrate solution (equivalent to $75 \mathrm{~g}$ anhydrous glucose) was administered orally to participants and serum glucose was measured 2 hours later. Fasting blood sugar (FBS) and 2-hour postglucose challenge ( $2 \mathrm{hPG}$ ) were measured on the day of blood collection by the enzymatic colorimetric method using glucose oxidase.

For lipid measurements, TC and triglyceride (TG) kits (Pars Azmoon Inc., Islamic Republic of Iran) were used. TC and TG were assayed using enzymatic colorimetric tests with cholesterol esterase and cholesterol oxidase, and glycerol phosphate oxidase, respectively. HDL-C was measured after precipitation of the apolipoprotein B containing lipoproteins with phosphotungistic acid. Low-density lipoprotein cholesterol (LDL-C) was calculated from serum TC, TG and HDL-C [17]; it was not calculated when serum TG concentration was greater than $400 \mathrm{mg} / \mathrm{dL}$. A lipid standard (Calibrator for Automated Systems, Boehringer Mannheim, Germany, cat. no. 759350) was used to calibrate the Selectra 2 autoanalyser for each day of laboratory analyses. All samples were analysed when internal quality control met the acceptable criteria. Inter- and intra-assay coefficients of variation were $2.0 \%$ and $0.5 \%$ for TC and $1.6 \%$ and $0.6 \%$ for $\mathrm{TG}$ respectively.

\section{Statistical analysis}

Statistical analyses were done using SPSS, version 10.01 statistical software package and data were presented as means and standard deviations (SD). Prevalence estimates of CHD were adjusted to the World Health Organization (WHO) world standard population distribution. Student $t$-test was used for comparison of means and chisquared tests were used for comparison of frequencies. $P$-values $<0.05$ were considered to be statistically significant.

Univariate analysis was performed using $\mathrm{CHD}$ as a dependent variable and age, sex, LDL-C, HDL-C, TC, TG, non-HDL-C, $\mathrm{LDL} / \mathrm{HDL}$ ratio, FBS, $2 \mathrm{hPG}$, hip and waist

المجلة الصحية لشرق المتوسط، منظمة الصحة العالمية، المجلد الخامس عشر، العدد (، 9 +. 
circumferences, weight, height, BMI, WHR, WHeR, SBP, DBP, hypertension, and smoking as independent variables. The independent variables that had $P$-values $<0.2$ on univariate analysis were considered for risk factor analysis in binary logistic regression analysis. Moreover, because of colinearity of some independent variables with others, we included just one of those with $r>0.60$. So the final variables for logistic regression analysis were: age, sex, SBP, DBP, FBS, 2hPG, hypertension, smoking, BMI, WHR, $\mathrm{LDL} / \mathrm{HDL}$ ratio, $\mathrm{TG}$ and $\mathrm{TC}$.

\section{Results}

Of all the 8071 individuals $\geq 30$ years who participated in the first phase of the Tehran Lipid and Glucose Study, information about angina pectoris, previous history of CHD and ECG-defined CHD was available for 5984 individuals.

Overall, after age adjustment, $21.8 \%$ of the Tehran population $\geq 30$ years had CHD, i.e. at least 1 of the 3 criteria (angina, previous history or ECG-defined CHD (Table $1)$. Women had marginally significantly higher prevalence than men $(22.3 \%$ versus $18.8 \%, P=0.05)$. Women also had higher prevalence of CHD based on angina or ECG definition alone $(21.3 \%$ versus $17.5 \%, P<$ $0.05)$.

There were 604 cases of angina (10.1\%), 324 cases with positive history of CHD (5.4\%) and 662 cases with ECG-defined CHD (11.1\%). After age adjustment, the prevalence of angina pectoris based on the Rose angina questionnaire was $10.7 \%$, self-reported history of CHD $6.0 \%$ and ECG-defined CHD 11.8\% (Figure 1).

Older subjects ( $\geq 65$ years) had a $62 \%$ higher prevalence of angina (15.8\% versus 9.7\%), had a more than 4 -fold higher prevalence of previous history of CHD (16.5\% versus $3.9 \%$ ) and had a 2.7 -fold higher

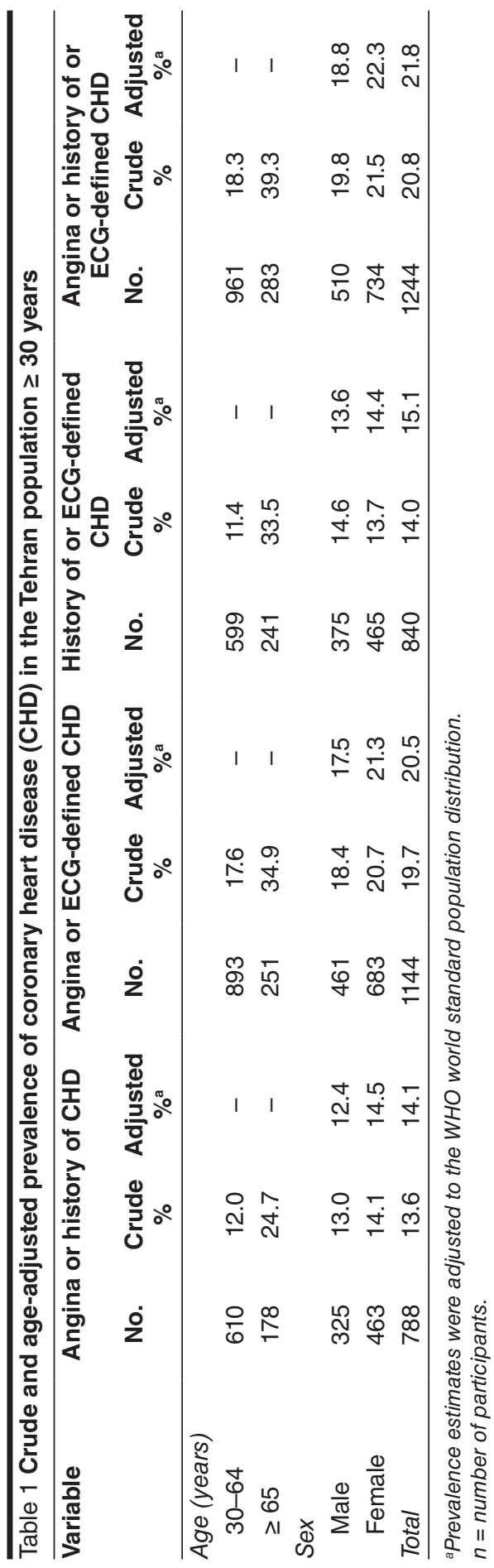


prevalence of ECG-defined CHD (24.9\% versus $9.2 \%)($ all $P<0.001)$.

After age adjustment, women had a significantly higher prevalence of angina pectoris than men $(11.5 \%$ versus $8.9 \%$, $P<0.001)$ and men had a higher rate of self-reported history of CHD than women $(6.5 \%$ versus $4.6 \%, P<0.01)$ (Figure 1$)$. There was no significant difference between men and women in the age-adjusted prevalence of ECG-defined CHD (probable and possible CHD) ( $11.5 \%$ versus $10.4 \%)$, but when the prevalence of probable CHD was compared between the sexes, it was significantly higher in males $(1.8 \%$ versus $0.9 \%, P=0.002$ ) (data not shown).

The descriptive statistics and the significance difference of clinical and biochemical characteristics for those with and without CHD (angina, previous history or ECGdefined CHD) are presented in Table 2. Subjects with CHD had significantly higher values of all the major CHD risk factors including age, BMI, SBP, DBP, FBS and $2 \mathrm{hPG}$, serum TC and TG, LDL and nonHDL cholesterol (all $P$ values $<0.001$ ). They also had greater waist circumference and higher WHR and WHeR $(P<0.001)$.

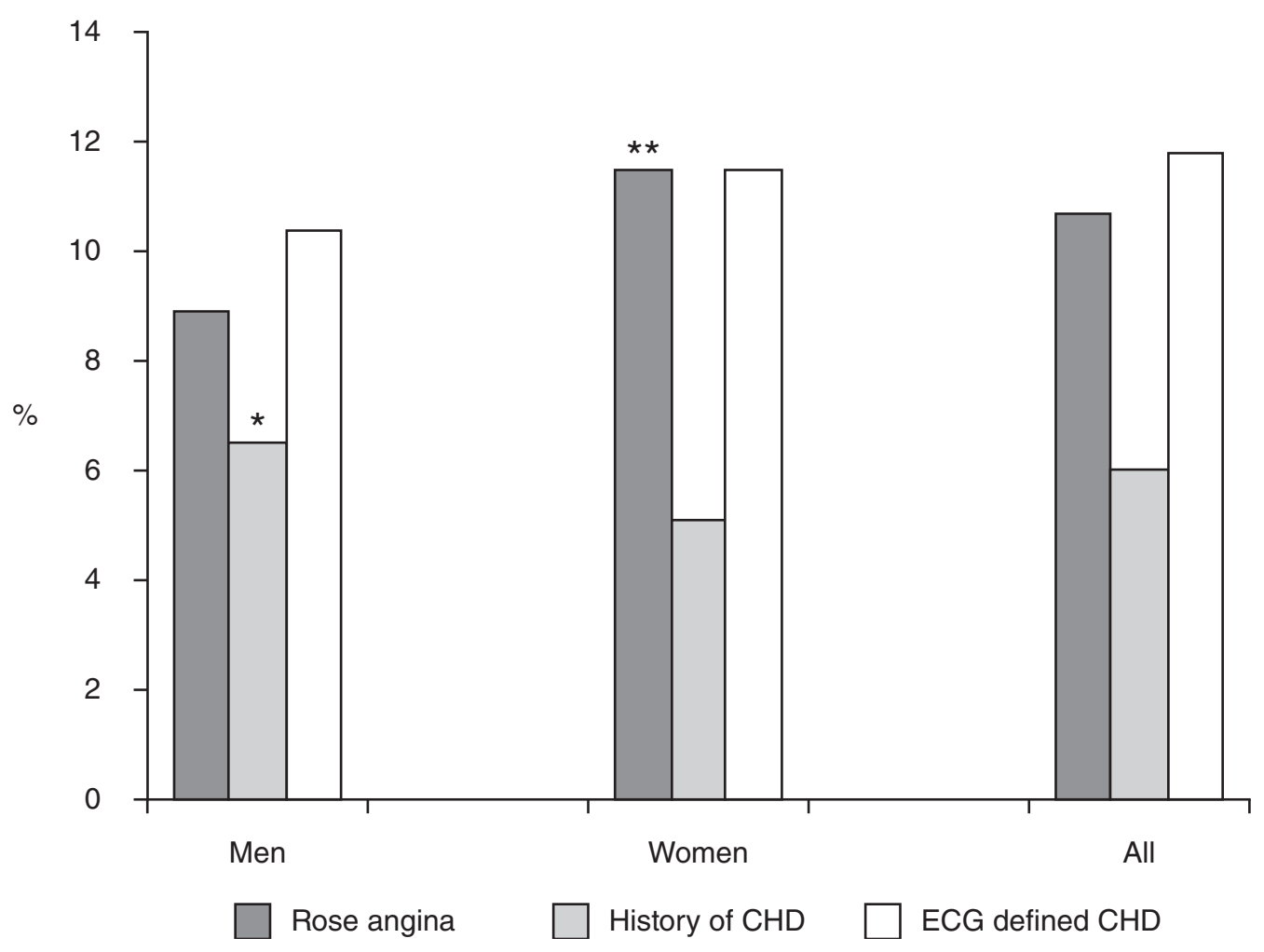

Figure 1 Age-adjusted prevalence of coronary heart disease (CHD) for the 3 different criteria (Rose angina, self-reported history of CHD and ECG-defined CHD) in the Tehran population $\geq 30$ years. Prevalence estimates were adjusted to the WHO world standard population distribution. ${ }^{*} P<0.01$; ${ }^{* \star} P<0.001$ versus opposite sex 
Table 2 Clinical and biochemical characteristics of the Tehran population $\geq 30$ years with and without coronary heart disease (CHD)

\begin{tabular}{lccc}
\hline Variable & $\begin{array}{c}\text { Without CHD } \\
(\boldsymbol{n}=\mathbf{4 7 4 0 )}\end{array}$ & $\begin{array}{c}\text { With CHDa } \\
(\boldsymbol{n}=\mathbf{1 2 4 4})\end{array}$ & $\boldsymbol{P}$-value \\
\hline Sex (female, \%) & 56.4 & 59.0 & 0.05 \\
Smoking (current and ex-smoker, \%) & 12.2 & 87.8 & 0.009 \\
Hypertension ( $\geq 140 / 90 \mathrm{mmHg}, \%)$ & 19.8 & 37.9 & $<0.001$ \\
& Mean (SD) & Mean (SD) & \\
Age (years) & $46(12)$ & $54(12)$ & $<0.001$ \\
Body mass index (kg/m²) & $27.3(4.5)$ & $28.2(4.6)$ & $<0.001$ \\
Waist circumference (cm) & $90(11)$ & $94(11)$ & $<0.001$ \\
Hip circumference (cm) & $101(9)$ & $102(10)$ & $<0.001$ \\
Waist-to-hip ratio & $0.88(0.08)$ & $0.91(0.08)$ & $<0.001$ \\
Waist-to-height ratio & $0.55(0.07)$ & $0.58(0.07)$ & $<0.001$ \\
Systolic blood pressure (mm Hg) & $120.2(18.5)$ & $130.4(23.5)$ & $<0.001$ \\
Diastolic blood pressure (mm Hg) & $78.6(10.5)$ & $81.4(12.1)$ & $<0.001$ \\
Fasting blood glucose (mg/dL) & $99.5(34.2)$ & $109.9(43.1)$ & $<0.001$ \\
2-hour plasma glucose (mg/dL) & $119.6(54.6)$ & $139.0(70.1)$ & $<0.001$ \\
Serum total cholesterol (mg/dL) & $214.9(44.0)$ & $228.4(50.4)$ & $<0.001$ \\
Serum triglycerides (mg/dL) & $180.6(116.1)$ & $200.1(123.8)$ & $<0.001$ \\
LDL cholesterol (mg/dL) & $137.9(37.6)$ & $148.3(42.7)$ & $<0.001$ \\
HDL cholesterol (mg/dL) & $41.9(10.7)$ & $41.7(11.1)$ & 0.4 \\
Non-HDL cholesterol (mg/dL) & $172.9(43.9)$ & $186.7(49.6)$ & $<0.001$ \\
LDL/HDL cholesterol & $3.4(1.2)$ & $3.7(1.3)$ & $<0.001$ \\
\hline
\end{tabular}

${ }^{a} \mathrm{CHD}$ was defined as Rose angina, self-reported history of $\mathrm{CHD}$, or ECG-defined CHD.

$n=$ number of participants; $S D=$ standard deviation; $L D L=$ low-density lipoprotein; $H D L=$ high-density lipoprotein.

Mean HDL cholesterol did not differ between subjects with and without $\mathrm{CHD}(P$ $=0.4$ ). The same results were obtained when the analysis was performed separately for different definitions of CHD (data not shown).

The prevalence of current or past smoking as well as hypertension was also significantly higher in subjects who had CHD than those who did not $(P<0.001)$ (data not shown).

A backwards conditional multiple logistic regression analysis was performed using CHD as the dependent variable and all statistically significant risk factors in the univariate analysis as independent variables. Table 3 shows the results of the final model. Six variables had an independent significant relationship with CHD: female $\operatorname{sex}(\mathrm{OR}=1.33 ; P<0.001)$, age $(\mathrm{OR}=1.03$; $P<0.001), 2 \mathrm{hPG}(\mathrm{OR}=1.002 ; P=0.011)$, $\mathrm{BMI}(\mathrm{OR}=1.02 ; P=0.014)$, WHR $(\mathrm{OR}=$ $3.20, P=0.040)$ and LDL/HDL cholesterol ratio $(\mathrm{OR}=1.09 ; P=0.001)$.

\section{Discussion}

In this community-based epidemiological study in Tehran, the overall age-standardized prevalence of CHD defined as positive history of CHD, angina or ischaemic ECG was $21.8 \%$. These results concur with previous reports of CHD prevalence in the 
Table 3 Independent determinants of coronary heart disease in the Tehran population $\geq \mathbf{3 0}$ years

\begin{tabular}{lcccr}
\hline Variable & $\boldsymbol{\beta}$ & SE & OR (95\% Cl) & \multicolumn{1}{c}{$\boldsymbol{P}$-value } \\
\hline Female sex & 0.290 & 0.091 & $1.33(1.11-1.59)$ & 0.001 \\
Age & 0.039 & 0.004 & $1.03(1.02-1.04)$ & $<0.001$ \\
Systolic blood pressure & 0.008 & 0.002 & $1.008(1.004-1.012)$ & $<0.001$ \\
Body mass index & 0.023 & 0.009 & $1.02(1.00-1.04)$ & 0.014 \\
Waist-to-hip ratio & 1.163 & 0.567 & $3.20(1.05-9.72)$ & 0.040 \\
2-hour plasma glucose & 0.002 & 0.001 & $1.002(1.000-1.003)$ & 0.011 \\
LDL/HDL cholesterol & 0.094 & 0.028 & $1.09(1.04-1.16)$ & 0.001 \\
\hline
\end{tabular}

$L D L=$ low-density lipoprotein; $H D L=$ high-density lipoprotein; $S E$ = standard error; $O R=$ odds ratio; $C l=$ confidence interval.

Results of multivariate conditional logistic regression analysis adjusted for sex, age, systolic and diastolic blood pressure, fasting and 2-hour plasma glucose, smoking, body mass index, waist-to-hip ratio, total cholesterol, triglycerides and $L D L / H D L$ cholesterol ratio.

Islamic Republic of Iran (Isfahan), which showed the overall prevalence of CHD based on the Rose questionnaire or ECG in subjects aged $30-79$ years to be $19.4 \%$, with a higher prevalence among women than men (21.9\% versus $16.0 \%, P<0.001)$ [ 18$]$. This is greater than other reports from the United States of America (USA) (11.8\%) [19], India (11\%) [20] and Saudi Arabia (5.5\%) [21]. Comparison of the results of these epidemiological studies is difficult because of differences in the criteria for defining CHD and in age distribution of the study population. We found only one study that used the same criteria as ours. In this study, based on the National Health and Nutrition Examination Survey data in the USA, the ageadjusted prevalence of CHD was $11.8 \%$. In a study in Kinmen, China, the overall prevalence of $\mathrm{CHD}$ in those aged $\geq 30$ years based on diagnostic ECG was $21 \%$, which is much higher than our results (11.8\%) and may be related to the long-term stress of the people who are living under military control in that region [22]. The high prevalence of CHD in Tehran residents may follow the high prevalence of metabolic syndrome and CVD risk factors in our population that has been reported previously $[8,9]$.
Women in the current study had a higher prevalence of Rose angina than men $(11.5 \%$ versus $8.9 \%$ ), which is similar to the results of other studies that found a higher rate of mild chest pain in women [23]. The reason for this higher positive response to the Rose questionnaire in women is not clear. However, the presence of a significant association between Rose angina and other CVD risk factors in our results, which contradict the findings of other studies [22], along with the higher mortality rate that has been reported in women with angina symptoms [24], suggests that the Rose questionnaire responses offer good evidence for the presence of CHD. In the current study, in multivariate regression analysis, female sex was significantly associated with CHD with an OR of 1.33. In a population-based study in India, the age-standardized prevalence of CHD based on previously documented myocardial infarction and positive ECG was significantly higher in females than males (12.4\% versus $5.0 \%, P<0.001)[20]$. The Kinmen study also reported the prevalence of ECG-defined CHD to be significantly higher in women than men $(25.4 \%$ versus $15.7 \%, P<0.001)[22]$. The higher prevalence of CHD in Iranian women reported in

المجلة الصحية لشرق المتوسط، منظمة الصحة العالمية، المجلد الخامس عشر، العدد (،9.ب. 
our study may follow the higher prevalence of metabolic syndrome in women compared with men ( $42 \%$ versus $24 \%, P<0.001)$ [9]. In the Islamic Republic of Iran, women generally have lower levels of physical activity than men, and overweight and obesity are more common among women [25].

In the current study $2 \mathrm{hPG}$ but not FBS was significantly associated with CHD. This implies, as in other reports [26], that $2 \mathrm{hPG}$ is more strongly associated with CVD risk and that this association is independent of FBS and other CVD risk factors.

We also found BMI and WHR to be independent predictors of CHD, but compared to BMI, WHR had a higher regression coefficient and OR in multiple logistic regression analysis. This is consistent with the results of a recent large cross-sectional study in a multiethnic population [27]. This showed that even after adjustment for other CVD risk factors, the OR of WHR for predicting myocardial infarction was significantly greater than that of BMI. These results may suggest that redefinition of obesity based on WHR instead of BMI increases the estimate of CHD attributable to obesity.

Our study showed the LDL/HDL cholesterol ratio to be an independent predictors of CHD. It was previously shown that the mean HDL-C level was low in the Tehran adult population (41 mg/dL) [28]. Regarding the high levels of LDL-C in the subjects with CHD, it is possible that in the presence of low HDL-C, even modest elevation of LDL-C with subsequent elevation of the LDL/HDL cholesterol ratio could contribute to CHD in our population. Similar results have also been reported in India [20].

The major limitation of this study was the cross-sectional assessment of CHD prevalence. Thus only survivors of CHD were assessed by this survey and the prevalence of non-fatal CHD does not reflect the burden of CHD in the Islamic Republic of Iran. Also the methods that were employed for diagnosis of CHD have well-recognized limited sensitivity and specificity and are expected to have even lower predictive values when used in the low-risk population [29]. Another limitation is that the data of the current study come from 1999-2001 and the situation in the Islamic Republic of Iran may have changed over this period. But bearing in mind the increasing trend in the prevalence of obesity (both general and abdominal obesity) [30] and the reported incidence rate of diabetes $(1 \%)$ in our population [31], it is likely that the prevalence of CHD reported in the current study has increased by now.

In conclusion, $21.8 \%$ of the Tehran population aged $\geq 30$ years was reported to have either angina pectoris, previous history of CHD or ECG findings consistent with a probable or possible CHD. These results demonstrate that CHD remains an important public health problem in Tehran residents. Regarding also the high prevalence of CVD risk factors and metabolic syndrome in this population, urgent steps are needed to reduce the risk factors by modifying diets and increasing levels of physical activity.

\section{References}

1. Castelli WP. Epidemiology of coronary heart disease: the Framingham study. American journal of medicine, 1984, 76(2A):4-12.

2. Keil U. Das weltweite WHO-MONICAProjekt: Ergebnisse und Ausblic [The worldwide WHO MONICA Project: results and perspectives]. Gesundheitswesen, 2005, 67(Suppl. 1):S38-45.

3. Thom TJ et al. Incidence, prevalence and mortality of cardiovascular disease in the United States. In: Fuster V et al., 
eds. Hurst's the heart, 9th ed. New York, McGraw-Hill, 1998.

4. Sytkowski PA et al. Sex and time trends in cardiovascular disease incidence and mortality: the Framingham heart study. American journal of epidemiology, 1996, 143(4):338-50.

5. Prevention and control of cardiovascular disease. Alexandria, World Health Organization Regional Office for the Eastern Mediterranean, 1995:24.

6. Zali M, Kazem M, Masjedi MR. [Health and disease in Iran]. Tehran, Islamic Republic of Iran, Deputy of Research, Ministry of Health, 1993 (Bulletin No. 10) [in Farsi].

7. Popkin BM, Gordon-Larsen P. The nutrition transition: worldwide obesity dynamics and their determinants. International journal of obesity and related metabolic disorders, 2004, 28(Suppl. 3):S2-9.

8. Prentice AM. The emerging epidemic of obesity in developing countries. International journal of epidemiology, 2006, 35(1):93-9.

9. Azizi F et al. Prevalence of metabolic syndrome in an urban population: Tehran Lipid and Glucose Study. Diabetes research and clinical practice, 2003, 61(1):29-37.

10. Azizi $\mathrm{F}$ et al. Cardiovascular risk factors in an Iranian urban population: Tehran Lipid and Glucose Study (Phase 1). Sozial-und präventivmedizin, 2002, 47(6):408-26.

11. Azizi $F$ et al. Distribution of blood pressure and prevalence of hypertension in Tehran adult population: Tehran Lipid and Glucose Study (TLGS), 1999-2000. Journal of human hypertension, 2002, 16(5):305-12.

12. Azizi $F$ et al. Tehran Lipid and Glucose Study: rationale and design. CVD prevention, 2000, 3:242-7.

13. Christiansen DH et al. Computer-assisted data collection in multicenter epidemio- logic research: the Atherosclerosis risk in Communities (ARIC) study. Controlled clinical trials, 1990, 11(2):101-15.

14. Rose G, McCartney P, Reid DD. Selfadministration of a questionnaire on chest pain and intermittent claudication. British journal of preventive and social medicine, 1977, 31(1):42-8.

15. Prineas RJ, Crow RS, Blackburn H. The Minnesota code manual of electrocardiographic findings: standards and procedures for measurement and classification. Boston, Wright-PSG, 1982.

16. Ford ES, Giles WH, Croft JB. Prevalence of nonfatal coronary heart disease among American adults. American heart journal, 2000, 139(3):371-7.

17. Friedewald WT, Levy RI, Fredrikson DS. Estimation of the concentration of lowdensity lipoprotein cholesterol in plasma, without use of the preparative ultracentrifuge. Clinical chemistry, 1972, 18(6):499502.

18. Sarraf-Zadegan $\mathrm{N}$ et al. The prevalence of coronary artery disease in an urban population in Isfahan, Iran. Acta cardiologica, 1999, 54(5):257-63.

19. Ford ES, Giles WH, Croft JB. Prevalence of nonfatal coronary heart disease among American adults. American heart journal, 2000, 139(3):371-7.

20. Mohan $\mathrm{V}$ et al. Prevalence of coronary artery disease and its relationship to lipids in a selected population in South India: The Chennai Urban Population Study (CUPS No. 5). Journal of the American College of Cardiology, 2001, 38(3):682-7.

21. Al-Nozha MM et al. Coronary artery disease in Saudi Arabia. Saudi medical journal, 2004, 25(9):1165-71.

22. Chen $\mathrm{CH}$ et al. Prevalence of coronary heart disease in Kin-Chen, Kinmen. International journal of cardiology, 1996, 55(1):87-95. 
23. Wilcosky T, Harris R, Weissfeld L. The prevalence and correlates of Rose Questionnaire angina among women and men in the Lipid Research Clinics Program Prevalence Study population. American journal of epidemiology, 1987, 125(3):400-9.

24. Owen-Smith V, Hannaford PC, Elliott AM. Increased mortality among women with Rose angina who have not presented with ischaemic heart disease. British journal of general practice, 2003, 53(495):784-9.

25. Mirmiran $P$ et al. [Measurement of total energy requirement in adults: prospective Tehran Lipid and Glucose Study]. Journal of Shaheed Beheshti University of Medical Sciences, 2001, 6:157-66 [in Farsi].

26. DECODE Study Group. Glucose tolerance and cardiovascular mortality: comparison of fasting and 2-hour diagnostic criteria. Archives of internal medicine, 2001, 161(3):397-405.

27. Yusef $S$ et al. Obesity and the risk of myocardial infarction in 27000 participants from 52 countries: a case-control study. Lancet, 2005, 366:1640-9.

28. Azizi $F$ et al. Determinants of serum HDL-C level in a Tehran urban population: the Tehran Lipid and Glucose Study. Nutrition, metabolism, and cardiovascular diseases, 2002, 12(2):80-9.

29. Simpson RJ Jr. Getting a handle on the prevalence of coronary heart disease. British heart journal, 1990, 64(5):291-2.

30. Azizi F, Azadbakht L, Mirmiran P. Trends in overweight, obesity and central fat accumulation among Tehranian adults between 1998-1999 and 2001-2002: Tehran Lipid and Glucose Study. Annals of nutrition and metabolism, 2005, 49(1):3-8.

31. Hadaegh $F$ et al. Waist/height ratio as a better predictor of type 2 diabetes compared to body mass index in Tehranian adult men-a 3.6-year prospective study. Experimental and clinical endocrinology and diabetes, 2006, 114(6):310-5. 